\title{
A period investigation of the overcontact binary system V417 Aquilae
}

\author{
Qian Shengbang ${ }^{1,2}$ \\ ${ }^{1}$ National Astronomical Observatories/Yunnan Observatory, Chinese Academy of Sciences, PO Box 110, 650011 Kunming, \\ PR China \\ 2 United Laboratory of Optical Astronomy, Chinese Academy of Science (ULOAC), 100012 Beijing, PR China
}

Received 4 November 2002 / Accepted 5 December 2002

\begin{abstract}
A detailed orbital period investigation of the short-period $(P=0.37)$ W UMa type star, V417 Aql, is presented based on the analysis of its $\mathrm{O}-\mathrm{C}$ data. It is shown that the period change of the binary system is continuous. A periodic variation, with a period of 42.4 years and an amplitude of 0.0130 , is found superimposed on a long-term period decrease $\left(\mathrm{d} P / \mathrm{d} t=-5.50 \times 10^{-8}\right.$ days/year). The period oscillation can be explained either by the light-time effect via the presence of an unseen third body or by magnetic activity cycles of the components. V417 Aql is a W-type overcontact binary system with a low mass ratio of $q=0.36$. The long-term period variation is in agreement with the conclusion of Qian (2001b) that a low-mass ratio W-type system usually shows a secular period decrease. This suggests that V417 Aql is on the AML-controlled stage of the evolutionary scheme proposed by Qian (2001b). Meanwhile, the light-curve paradox encountered by TRO theory is discussed.
\end{abstract}

Key words. stars: binaries: close - stars: binaries: eclipsing - stars: individual: V417 Aql - stars: evolution

\section{Introduction}

The light variability of V417 Aql (BD + $054202=$ GSC4904531 = PPM168201) was discovered by Hoffmeister (1935). It was subsequently classified as W UMa type binary by Soloviev (1937) who gave four times of light minimum and determined a period of 0.370114 . Further works by Soloviev (1949) and Kramer (1947) yielded improved periods of 0.3701207 and $0 \mathrm{~d} 3701250$, respectively. A $B-V$ color index of 0.62 obtained by Koch (1974) indicates that it is a solar type star. Normal complete photoelectric light curves in $B$ and $V$ were published by Faulkner (1983) who also gave a linear ephemeris: Min.I $(H J D)=2445554.7241+0.3703072 \times E$. Recently, high-precision photometric light curves (in $U, B$, and $V$ ) were published by Samec et al. (1997). They gave solutions of geometric elements with the Wilson-Devinney (W-D) method. It was shown that V417 Aql is a W-type overcontact binary system (the Roche lobe fill-out factor is $f=19 \%$ ) with a low mass ratio of $q_{\mathrm{ph}}=0.368$. The mass ratio is the same as the spectroscopic one $\left(q_{\mathrm{sp}}=0.362\right)$ published by Lu \& Rucinski (1999). By combining the photometric elements with the spectroscopic ones, absolute parameters of the system were determined by Lu \& Rucinski (1999).

Linear light elements of the system have been given by several authors (e.g., see Table 1). Kämper (1984) published

Send offprint requests to: Qian Shengbang,

e-mail: qsb@netease.com
17 times of light minimum and presented the first period study of the binary system. The recent period studies by Samec et al. (1997) and Pauley et al. (1996) showed that two constant periods before HJD 2433000 and for modern era were connected by a continuous but smooth period decrease taking place over 24 years. Note, however, that the ephemeris in Eq. (2) of the paper by Samec et al. (1997), based on the recent minima, is misprinted and should read Min.I $(H J D)=2449546.4979+$ $0.3703119 \times E$, as in Pauley et al. (1996). In order to understand the evolutionary state of V417 Aql and to look for cyclic variations in the orbital periods of overcontact binary systems, the properties of period changes of V417 Aql are investigated in detail.

\section{Variations of the $\mathrm{O}-\mathrm{C}$ curve}

Times of light minimum of V417 Aql were collected by Samec et al. (1997), kindly provided by Dr. R. D. Samec. After their compilation, some photoelectric or CCD eclipse times were published (see Table 2). Although the following linear ephemeris:

Min. $I=2449546.4982+0.3703148 \times E$

given by Samec et al. (1997) was misprinted, it was used to calculate the $\mathrm{O}-\mathrm{C}$ values of all the times of minimum light. The corresponding $(\mathrm{O}-\mathrm{C})$ diagram is shown in Fig. 1 where 
Table 1. Linear light elements for V417 Aql.

\begin{tabular}{lll}
\hline \hline$T_{0}$ (days) & $P_{0}$ (days) & References \\
\hline 2428427.127 & 0.370114 & Soloviev (1937) \\
2428426.933 & 0.3701250 & Kramer (1947) \\
2428427.127 & 0.3701207 & Soloviev (1949) \\
2428427.127 & 0.3701251 & Koch (1956) \\
2445554.7241 & 0.3703072 & Faulkner (1983) \\
2428427.126 & 0.3703135 & Agerer (1984) \\
2428427.122 & 0.3703146 & Agerer (1984) \\
2445554.7240 & 0.3703110 & Kämper (1984) \\
2428427.126 & 0.3703135 & Kämper (1984) \\
2445554.7240 & 0.37031142 & Faulkner (1983) \\
2449546.4979 & 0.3703119 & Pauley et al. (1996) \\
2449546.4982 & 0.3703148 & Samec et al. (1997) \\
2449546.4983 & 0.37031178 & the present paper \\
\hline
\end{tabular}

circles denote visual or photographic (VP) data and dots represent photoelectric or charge-coupled device (CCD) observations. As plotted in Fig. 1, the $\mathrm{O}-\mathrm{C}$ values of the VP data show slightly large scatter (up to $0 \mathrm{~d} 02$ ). The $\mathrm{O}-\mathrm{C}$ residuals of all photoelectric or CCD observations are listed in Table 2. Based on those data, a new linear ephemeris:

Min.I $=2449546.4983(3)+0.37031178(7) \times E$

is derived, which can be used to predict the future times of light minimum.

The period studies by Samec et al. (1997) and Pauley et al. (1996) indicate that, after HJD 2433000, a continuous period decrease took place over 24 years, and subsequently the period of V417 Aql has been constant during the modern era. However, the recent data shown in Fig. 1 (solid dots) reveal that the period is not constant, but changes continuously. This suggests that the period of V417 Aql varies in a continuous way over the 64-year time interval since its discovery, and its change is very complex. As one can see in Fig. 1, a downward parabolic curve (dashed line) cannot give a good description of the general trend of the $\mathrm{O}-\mathrm{C}$ curve, indicating that there may exist a periodic variation superimposed on a secular period decrease. Therefore, a sinusoidal term is added to a quadratic ephemeris to give a good fit to the data points (solid line in Fig. 1). With weights 8 to photoelectric or CCD observations and 1 to VP data, a weighted least-squares solution leads to the following ephemeris:

$$
\begin{aligned}
\text { Min.I }= & 2449546.5096( \pm 0.0012) \\
& +0^{\mathrm{d}} 37031273( \pm 0.00000019) \times E \\
& -2.79( \pm 0.28) \times 10^{-11} \times E^{2} \\
& +0.0130( \pm 0.0013) \\
& \times \sin \left[0^{\circ} .0086 \times E+259^{\circ} .9\left( \pm 7^{\circ} .0\right)\right] .
\end{aligned}
$$

The value of the sum of the weighted squares of the residuals from Eq. (3) is $\Sigma=0.0040$, much smaller than that from the quadratic part of the equation $(\Sigma=0.041)$. This suggest that this ephemeris can fit the general $\mathrm{O}-\mathrm{C}$ trend very well. With the quadratic term of this ephemeris, a continuous period decrease, at rate of $\mathrm{d} P / \mathrm{d} t=-5.50 \times 10^{-8}$ days/year, is determined. The sinusoidal term in Eq. (3) reveals a periodic change with an

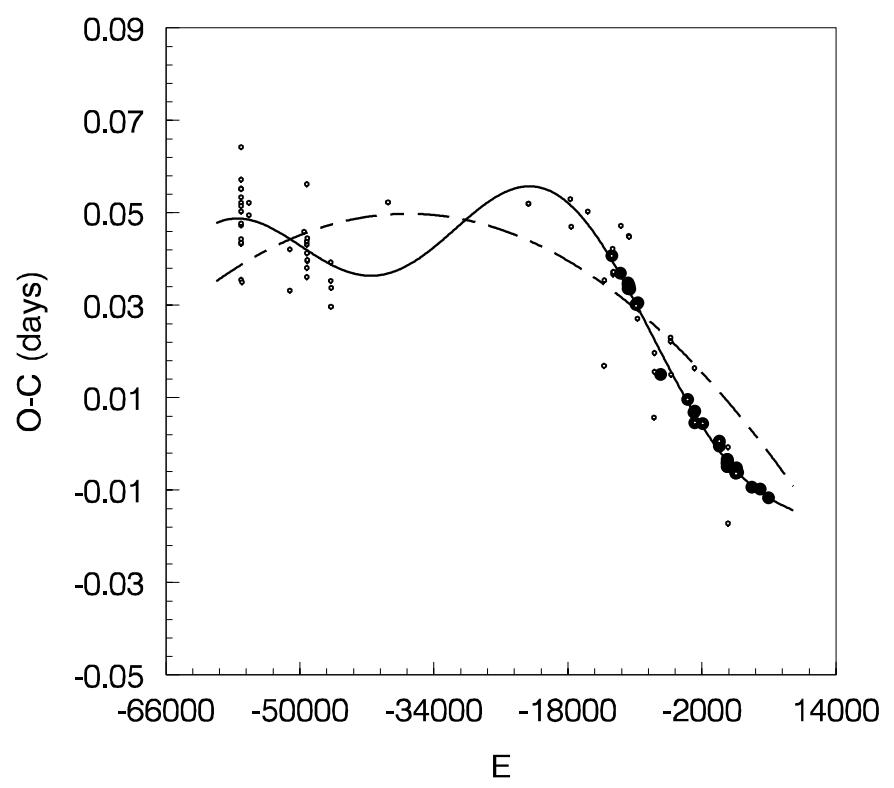

Fig. 1. O-C curve of the short-period W UMa type binary V417 Aql computed with Eq. (1). Also given in the figure are the quadratic fit (dashed line) and its description by a combination of a quadratic and a periodic ephemerides (solid line). Circles refer to visual or photographic observations and dots to photoelectric or CCD data.

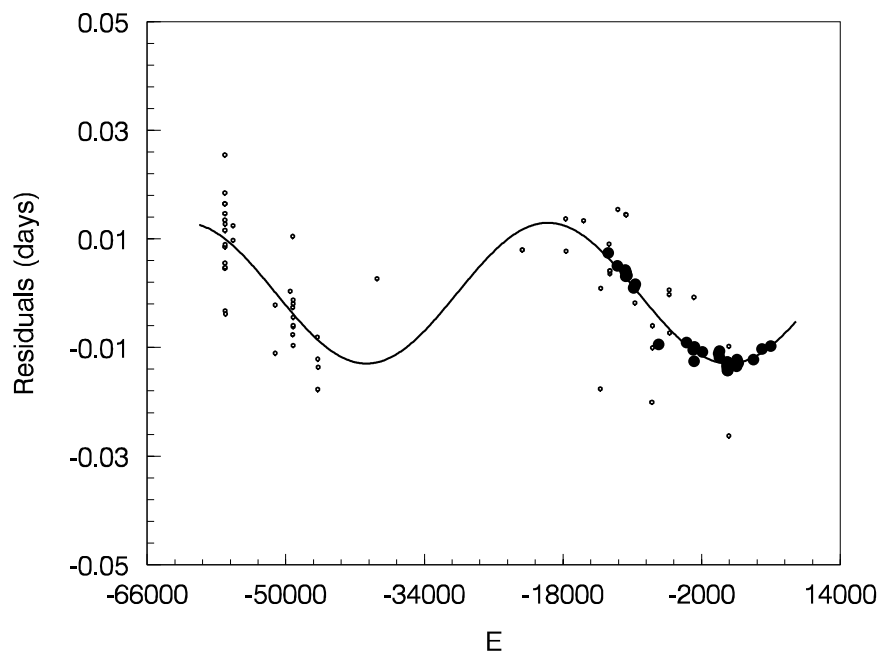

Fig. 2. Residuals of V417 Aql based on the quadratic part of Eq. (3) and their description by the sinusoidal term (solid line). Symbols are the same as in Fig. 1.

amplitude of 0.0130 and a period of 42.4 years. This is more easily seen from Fig. 2 where the residuals $\left[(\mathrm{O}-\mathrm{C})^{\prime}\right.$ values] calculated from the quadratic part of Eq. (3) were shown. The solid line in the figure represents their fit by the sinusoidal term.

\section{Discussions and conclusions}

By the analysis of the O-C curve of V417 Aql, a periodic change is found to be superimposed on a long-term period decrease $\left(\mathrm{d} P / \mathrm{d} t=-5.50 \times 10^{-8}\right.$ days/year $)$. A period oscillation superimposed on a secular term is usually encountered for W UMa type binary stars. Other examples are V566 Oph 
Table 2. Photoelectric and CCD times of light minimum for V417 Aql.

\begin{tabular}{lllllll}
\hline \hline JD (Hel.) & Method & Minimum & $E$ & O-C & $(\mathrm{O}-\mathrm{C})^{\prime}$ & Ref. \\
$2400000+$ & & & & & & \\
\hline 44815.3970 & pe & I & -12776 & +0.0407 & +0.0076 & $(1)$ \\
45196.4471 & pe & I & -11747 & +0.0369 & +0.0052 & $(2)$ \\
45542.6894 & pe & I & -10812 & +0.0348 & +0.0044 & $(3)$ \\
45550.6508 & pe & II & -10790.5 & +0.0344 & +0.0041 & $(3)$ \\
45554.7234 & pe & II & -10779.5 & +0.0336 & +0.0033 & $(3)$ \\
45575.6468 & pe & I & -10723 & +0.0342 & +0.0040 & $(3)$ \\
45605.6416 & pe & I & -10642 & +0.0335 & +0.0034 & $(3)$ \\
45892.4470 & pe & II & -9867.5 & +0.0301 & +0.0011 & $(4)$ \\
45935.4036 & pe & II & -9751.5 & +0.0302 & +0.0014 & $(5)$ \\
45962.6221 & pe & I & -9678 & +0.0305 & +0.0018 & $(6)$ \\
46977.4543 & pe & II & -6937.5 & +0.0150 & -0.0093 & $(7)$ \\
48163.3820 & CCD & I & -3735 & +0.0096 & -0.0090 & $(8)$ \\
48448.5217 & pe & I & -2965 & +0.0069 & -0.0103 & $(9)$ \\
48490.3650 & CCD & I & -2852 & +0.0046 & -0.0124 & $(10)$ \\
48500.3660 & pe & I & -2825 & +0.0071 & -0.0098 & $(9)$ \\
48843.4600 & CCD & II & -1898.5 & +0.0044 & -0.0107 & $(11)$ \\
49546.4979 & pe & I & 0 & +0.0003 & -0.0110 & $(12)$ \\
49568.5314 & pe & II & 59.5 & -0.0005 & -0.0117 & $(12)$ \\
49571.4950 & pe & II & 67.5 & +0.0006 & -0.0106 & $(13)$ \\
49917.9200 & pe & I & 1003 & -0.0039 & -0.0131 & $(14)$ \\
49918.8454 & pe & II & 1005.5 & -0.0043 & -0.0135 & $(14)$ \\
49919.9563 & pe & II & 1008.5 & -0.0044 & -0.0136 & $(14)$ \\
49920.6964 & pe & II & 1010.5 & -0.0049 & -0.0141 & $(14)$ \\
49920.8826 & pe & I & 1011 & -0.0039 & -0.0131 & $(14)$ \\
49921.4385 & pe & II & 1012.5 & -0.0034 & -0.0126 & $(15)$ \\
49921.8081 & pe & II & 1013.5 & -0.0041 & -0.0133 & $(14)$ \\
49922.9183 & pe & II & 1016.5 & -0.0049 & -0.0141 & $(14)$ \\
50303.4155 & pe & I & 2044 & -0.0062 & -0.0132 & $(16)$ \\
50312.4880 & CCD & II & 2068.5 & -0.0064 & -0.0133 & $(17)$ \\
50315.4511 & pe & II & 2076.5 & -0.0058 & -0.0127 & $(16)$ \\
50315.4517 & pe & II & 2076.5 & -0.0052 & -0.0121 & $(16)$ \\
51016.4534 & pe & II & 3969.5 & -0.0094 & -0.0121 & $(18)$ \\
51378.4357 & pe & I & 4947 & -0.0098 & -0.0102 & $(19)$ \\
51747.4526 & pe & II & 5943.5 & -0.0116 & -0.0097 & $(20)$ \\
\hline & & & & & & \\
& & & & & \\
450
\end{tabular}

References in Table 3:

(1) BBSAG Bull. 56; (2) Fernandes (1983); (3) Faulkner (1983); (4) BBSAG Bull. 73; (5) Agerer (1984); (6) Faulkner (1986); (7) Agerer (1988); (8) BBSAG Bull. 96; (9) Agerer (1992); (10) BBSAG Bull. 99; (11) BBSAG bull. 102; (12) Agerer \& Hubscher (1995); (13) BBASG Bull. 102; (14) Samec et al. (1997); (15) Agerer \& Hubscher (1996); (16) Agerer \& Hubscher (1997); (17) BBASG Bull. 114; (18) Agerer \& Hubscher (1999); (19) Agerer \& Hubscher (2001); (20) Agerer \& Hubscher (2002).

(Qian 2001a), V502 Oph (Derman \& Demircan 1992), U Peg (Zhai et al. 1984), AB And (Borkovits \& Hegedues 1996; Kalimeris et al. 1994), YY Eri (Kim et al. 1997), RW Com (Qian 2002), SW Lac (Pribulla et al. 1999), CK Boo (Qian \& Liu 2000), V839 Oph (Wolf et al. 1996), and V700 Cyg (Qian 2003). These periodic variations are usually explained either by the light-time effect via the presence of a third body or by magnetic activity cycles in both components, because they are fast-rotating solar-type stars.

For V417 Aql, if the period oscillation is a result of the light-time effect via the presence of an additional third body, with the semi-amplitude of the $\mathrm{O}-\mathrm{C}$ oscillation, the value $a_{12}^{\prime} \sin i^{\prime}$ is computed to be: $a_{12}^{\prime} \sin i^{\prime}=2.25( \pm 0.23) \mathrm{AU}$. Then using the following equation:

$f(m)=\frac{\left(M_{3} \sin i^{\prime}\right)^{3}}{\left(M_{1}+M_{2}+M_{3}\right)^{2}}=\frac{4 \pi^{2}}{G T_{3}^{2}} \times\left(a_{12}^{\prime} \sin i^{\prime}\right)^{3}$, a small mass function $f(m)=0.0064( \pm 0.0019) M_{\odot}$ is determined for the third body. Taking the absolute parameters given by Lu \& Rucinski (1999), $M_{1}=1.4 M_{\odot}$ and $M_{2}=0.5 M_{\odot}$, the values of the masses and the orbital radii of the third body for several different values of $i^{\prime}$ are computed and are shown in Table 3. If we assume that the third body is coplanar to the orbit of the eclipsing pair (i.e., $i^{\prime}=84.47^{\circ}$ ), the values of the mass and the orbital radius of the third body should be: $m_{3}=0.316 M_{\odot}$ and $a_{3}=16.12 \mathrm{AU}$, respectively. In this case, the supposed third body is very difficult to detect because its luminosity is extremely small. Since no third light was reported in the photometric study of Samec et al. (1997) and no spectral lines of a third body were found by Lu \& Rucinski (1999), the orbital inclination of the third body should not be extremely low, i.e., $i^{\prime}>30^{\circ}$.

V417 Aql consists of G0 V and F9 V spectral type stars (Samec et al. 1997) that should show strong magnetic activity. 
Table 3. The values of the mass and the orbital radii of the assumed third body in V417 Aql.

\begin{tabular}{lll}
\hline \hline Parameters & Values & Units \\
\hline$A$ & $0.0130( \pm 0.0013)$ & days \\
$T_{3}$ & $42.4($ assumed $)$ & years \\
$a_{12}^{\prime} \sin i^{\prime}$ & $2.25( \pm 0.23)$ & AU \\
$f(m)$ & $6.4( \pm 1.9) \times 10^{-3}$ & $M_{\odot}$ \\
$m_{3}\left(i^{\prime}=90^{\circ}\right)$ & $0.31( \pm 0.06)$ & $M_{\odot}$ \\
$m_{3}\left(i^{\prime}=70^{\circ}\right)$ & $0.34( \pm 0.06)$ & $M_{\odot}$ \\
$m_{3}\left(i^{\prime}=50^{\circ}\right)$ & $0.42( \pm 0.07)$ & $M_{\odot}$ \\
$m_{3}\left(i^{\prime}=30^{\circ}\right)$ & $0.70( \pm 0.10)$ & $M_{\odot}$ \\
$m_{3}\left(i^{\prime}=10^{\circ}\right)$ & $3.13( \pm 0.54)$ & $M_{\odot}$ \\
$a_{3}\left(i^{\prime}=90^{\circ}\right)$ & $16.2( \pm 3.4)$ & $\mathrm{AU}$ \\
$a_{3}\left(i^{\prime}=70^{\circ}\right)$ & $15.7( \pm 3.2)$ & $\mathrm{AU}$ \\
$a_{3}\left(i^{\prime}=50^{\circ}\right)$ & $14.5( \pm 2.7)$ & $\mathrm{AU}$ \\
$a_{3}\left(i^{\prime}=30^{\circ}\right)$ & $12.7( \pm 2.2)$ & $\mathrm{AU}$ \\
$a_{3}\left(i^{\prime}=10^{\circ}\right)$ & $7.91( \pm 1.6)$ & $\mathrm{AU}$ \\
\hline
\end{tabular}

The period oscillation can also be explained as a consequence of possible magnetic cycles in both components (Applegate 1992; Lanza et al. 1998). If this is in the case, by inserting the physical parameters, $M_{1}=1.4 M_{\odot}, M_{2}=0.5 M_{\odot}$, and $a=2.68 R_{\odot}$, given by Lu \& Rucinski (1999), in the following equation:

$\frac{\Delta P}{P}=-9\left(\frac{R}{a}\right)^{2} \frac{\Delta Q}{M R^{2}}$,

the required variation of the quadruple moments $\Delta Q$ in order to reproduce the period oscillation are calculated to be $\Delta Q=5.93 \times 10^{49}$ and $1.98 \times 10^{49} \mathrm{~g} . \mathrm{cm}^{2}$ for the primary and the secondary component, respectively. However, V417 Aql is an overcontact binary system where both components share a common convective envelope (CCE) $(f=19 \%)$. How this mechanism works in this kind of systems is not clear.

Recently, based on long-term period changes of $30 \mathrm{~W}$ type W UMa-type stars, a possible relation between the period change and the mass ratio was found by Qian (2001b). It is shown that the period change of a high-mass ratio system is usually increasing, while that of a low-mass ratio one is decreasing. The photometric and the spectroscopic investigations by Samec et al. (1997) and Lu \& Rucinski (1999) indicate that V417 Aql is a W-type overcontact binary system with a low-mass ratio $(q=0.36)$. The long-term period decrease of V417 Aql is in agreement with the conclusion of Qian (2001b). If the secular period decrease is caused by a conservative mass transfer (with no magnetic effect) from the primary to the secondary component, then using the following well-known equation:

$\dot{P} / P=3\left(M_{1} / M_{2}-1\right) \Delta M_{1} / M_{1}$,

the mass transfer rate is estimated to be: $\mathrm{d} M / \mathrm{d} t=1.54 \times$ $10^{-7} M_{\odot} /$ year.

The thermal relaxation oscillation (TRO) theory of overcontact binaries was orignally proposed by Lucy (1976) and Flannery (1976). By assuming a conservation of total mass and angular momentum, it predicted that an overcontact binary system should evolve in thermal cycles around a marginal overcontact. In the broken overcontact stage, the system looks like a semi-detached binary with a lobe-filling primary and showing a $\beta$ Lyrae-type light curve. Thus systems with $\beta$ Lyraetype light curves should exist within the period range of the W UMa stars. The paucity of short-period $(P<0.4)$ binary systems with non-W UMa light curves was called as "lightcurve paradox". Although a detailed computation by Robertson \& Eggleton (1977) indicated that this shortcoming of the TRO model can be removed because the fraction of time spent in the broken stage is very small for low-mass ratio $\mathrm{W}$ UMa stars, many investigations, especially the more recent one by Hazlehurst (2001), reveals that TRO theory cannot resolve the so-called light-curve paradox.

Long-term period changes, both decreasing and increasing, are common for W UMa-type stars. In order to explain these properties, Qian (2001b) pointed out that the dynamic evolution of overcontact binaries may be TRO plus variable AML (angular momentum loss) via the change of the depth of overcontact. The main assumption of the evolutionary scheme is that the angular momentum loss (AML) rate change with the variation of the depth of overcontact, supported by the X-ray, EUV and other observations (see Qian 2001b, 2003). When a W UMa-type star shows a long-term period decrease, the CCE will become deeper and the increased mixing may result in a lower AML rate (see also Vilhu 1981; Smith 1981). Thus the period will increase and cause a shallower CCE. Magnetic activity will be stronger and a higher AML rate is expected. Finally, the period will decrease again and the system will oscillate around a critical mass ratio. A broken phase predicted by TRO theory connot arise. Therefore, a short-period $(P<0.4)$ close binary with a $\beta$ Lyrae type light curve can not be observed and the light-curve paradox encountered by the TRO model can be removed. The long-term period decrease of V417 Aql may suggest that it is on the AML-controlled stage of this evolutionary scenario.

Acknowledgements. I would like to thank the referee Dr. G. Torres for a careful reading of this paper and valuable comments and suggestions. I am indebted to Dr. Ronald G. Samec who sent me the times of light minimum of the eclipsing binary system collected in their paper $(1999, \mathrm{AJ} 113,401)$ and to the many observers, amateur and professional, who observed the wealth of data on the eclipsing binary star. This work is partly supported by Chinese Natural Science Foundation (No. 100030004), Yunnan Natural Science Foundation (No. 2001A0026Q), and by National Key Fundamental Research Project through grant G1999075405.

\section{References}

Agerer, F. 1984, BAV Rundbrief, 33, 4

Agerer, F. 1988, BAV Mitt., No. 50

Agerer, F. 1984, BAV Mitt., No. 60

Agerer, F., \& Hubscher, J. 1995, IBVS, 4222

Agerer, F., \& Hubscher, J. 1996, IBVS, 4383

Agerer, F., \& Hubscher, J. 1997, IBVS, 4472

Agerer, F., \& Hubscher, J. 1999, IBVS, 4711

Agerer, F., \& Hubscher, J. 2001, IBVS, 5016

Agerer, F., \& Hubscher, J. 2002, IBVS, 5296

Applegate, J. H. 1992, APJ, 385, 621

Borkovits, T., \& Hegedues, T. 1996, A\&AS, 120, 63

Derman, E., \& Demircan, O. 1992, AJ, 103, 1658 
Faulkner, D. R. 1983, IBVS, 2439

Faulkner, D. R. 1986, PASP, 98, 690

Fernandes, M. 1983, BAV Mitt., No. 36

Flannery, B. P. 1976, APJ, 205, 317

Hazlehurst, J. 2001, The Observatory, 121, 86

Hoffmeister, C. 1935, Astr. Nach., 255, 403

Kämper, B.-C. 1984, BAV Rundbrief, 33, 152

Kalimeris, A., Rovithis-Livaniou, H., Rovithis, P., et al. 1994, A\&A, 291,765

Kim, C. H., Jeong, J. H., Demircan, O., Muyesseroglu, Z., \& Budding, E. 1997, AJ, 114, 2753

Koch, R. H. 1956, AJ, 56, 47

Koch, R. H. 1974, AJ, 79, 34

Kramer, E. N. 1947, Kazan Astr. Circ., No. 66 Lanza, A. F., Rodonò, M., \& Rosner, R. 1998, MNRAS, 296, 893

Lu, W. X., \& Rucinski, S. M. 1999, AJ, 118, 515

Lucy, L. B. 1976, APJ, 205, 208
Pauley, B., Carrigan, B., French, J., Looi, M., \& Samec, R. G. 1996, IBVS, 4358

Pribulla, T., Chochol, D., \& Parimueha, S. 1999, Contrib. Astron. Obs. Skainate Pleso., 29, 111

Qian, S. B. 2001a, MNRAS, 328, 914

Qian, S. B. 2001b, MNRAS, 328, 635

Qian, S. B. 2002, A\&A, 384, 908

Qian, S. B. 2003, MNRAS, in press

Qian, S. B., \& Liu, Q. Y. 2000, ApSS, 271, 331

Robertson, J. A., \& Eggleton, P. P. 1977, MNRAS, 179, 359

Samec, R. G., Pauley, B. R., \& Carrigan, B. J. 1997, AJ, 113, 401

Smith, R. C. 1984, Q. J1 R. Astro. Soc., 25, 405

Soloviev, A. 1937, Tadjik Obs. Circ., No. 22

Soloviev, A. 1949, Var. Stars, 6, 287

Vilhu, O. 1981, ApSS, 78, 401

Wolf, M., Sarounová, L., \& Molík, P. 1996, IBVS, 4304

Zhai, D. S., Leung, K. C., \& Zhang, R. X. 1984, A\&AS, 57, 487 